\title{
S100A7 promotes the migration and invasion of osteosarcoma cells via the receptor for advanced glycation end products
}

\author{
KEN KATAOKA* , TOMOYUKI ONO* , HITOSHI MURATA, MIKA MORISHITA, \\ KEN-ICHI YAMAMOTO, MASAKIYO SAKAGUCHI and NAM-HO HUH \\ Department of Cell Biology, Okayama University Graduate School of Medicine, \\ Dentistry and Pharmaceutical Sciences, Okayama 700-8558, Japan
}

Received December 14, 2011; Accepted February 1, 2012

DOI: $10.3892 / 01.2012 .612$

\begin{abstract}
Osteosarcoma is the most common malignant tumor of bone in childhood and adolescence. Despite intensive research for new therapies, the outcome in patients with metastasis remains extremely poor. S100 proteins are involved in the proliferation, cell cycle progression and metastasis of numerous malignant tumors, including osteosarcoma. In the present study, we identified S100A7 as a candidate to promote the migration of osteosarcoma cells. S100A7 promoted the migration and invasion of osteosarcoma cells as assayed in vitro. An in vitro pull-down assay revealed the binding of the recombinant S100A7 protein with its putative receptor, the receptor for advanced glycation end products (RAGE). The downregulation of RAGE by a specific siRNA markedly suppressed the migration and invasion of osteosarcoma cells. Furthermore, the matrix metalloproteinase activity of osteosarcoma cells was enhanced by S100A7 and suppressed by the downregulation of RAGE. These results indicate that S100A7 promotes the migration and invasion of osteosarcoma cells through RAGE. The S100A7-RAGE axis may thus be a new target for preventing the invasion and/or metastasis of osteosarcoma.
\end{abstract}

\section{Introduction}

Osteosarcoma is the most common malignant tumor of bone in childhood and adolescence (1). It is an aggressive tumor that metastasizes primarily to the lung. Clinical efforts, including the development of protocols for effective chemotherapy, have

Correspondence to: Dr Ken Kataoka, Department of Cell Biology, Okayama University Graduate School of Medicine, Dentistry and Pharmaceutical Sciences, 2-5-1 Shikatacho, Kita-ku, Okayama 700-8558, Japan

E-mail: kenk@md.okayama-u.ac.jp

${ }^{*}$ Contributed equally

Abbreviations: RAGE, receptor for advanced glycation end products; siRNA, small interfering RNA; CBB, Coomassie brilliant blue; MMP, matrix metalloproteinase

Key words: osteosarcoma, S100A7, RAGE, migration, invasion significantly improved the 5-year survival rate of osteosarcoma patients from 15 to $70 \%$ (2). However, $30-40 \%$ of patients still succumb to the disease, mainly due to distant metastasis to the lung (3). Despite intensive research for new therapies, the outcome in patients with metastasis remains extremely poor.

The S100 protein family consists of 20 calcium-binding proteins with EF hand motifs (4). S100 proteins have been shown to have intracellular and extracellular roles in the regulation of diverse processes, including protein phosphorylation, cell growth and motility, cell-cycle regulation, transcription, differentiation and cell survival (5). S100 proteins are normally expressed in a tissue-specific manner and their dysregulation has been causally linked to numerous diseases, including several types of cancer (6). For example, S100A4 is involved in proliferation, cell cycle progression and the metastasis of a number of malignant tumors, including osteosarcoma (7). In a screening study for S100 proteins potentially involved in the migration of osteosarcoma cells, we identified S100A7 as a candidate.

S100A7 (Psoriasin) was originally identified as a protein that is upregulated in psoriatic skin (8). Several S100 proteins, including S100A7, S100A8/9, S100A12 and S100A15 (koebnerisin) were found to be upregulated in psoriatic skin $(4,9)$ and these S100 proteins are localized within the epidermal differentiation complex (EDC) on human chromosome 1 (1q21) (10). Among the S100 family genes in the EDC, S100A7 and S100A15 are almost identical in sequence $(<90 \%)$. Despite their similarity, S100A7 and S100A15 are distinct in tissue distribution, regulation and function, being exemplary for the diversity within the S100 family (9). In skin tissue, S100A7 interacts with epidermal fatty acid-binding protein and the expression of S100A7 is elevated under pathological conditions, including inflammation and hyperproliferation (4). Wolf et al reported that S100A7 acts through its putative receptor, the receptor for advanced glycation end products (RAGE), in the inflammatory epidermis (9). However, the roles of S100A7 in other biological/pathological contexts, including its effects on tumor cells, remain unclear.

In the present study, we investigated the effects of S100A7 on the migration and invasion of osteosarcoma cells.

\section{Materials and methods}

Cell culture. The human osteosarcoma cell line Saos-2 (11) and the human breast cancer cell line MCF-7 (12) were obtained 
from the American Type Culture Collection (Rockville, MD, USA). The normal human fibroblast cell line OUMS-24 (13) and the human osteosarcoma cell line $\mathrm{HuO} 9$ (14) were established in our department. Saos-2, MCF-7 and HuO9 were cultured in RPMI-1640 (Invitrogen, Carlsbad, CA, USA). OUMS-24 was maintained in Dulbecco's modified EM (Nissui, Tokyo, Japan). All the media were supplemented with 10\% FBS (Invitrogen), $100 \mu \mathrm{g} / \mathrm{ml}$ kanamycin (Meiji Seika, Tokyo, Japan) and $0.5 \mu \mathrm{g} / \mathrm{ml}$ Fungizone (Invitrogen). Primary normal human keratinocytes (NHK) were purchased from Kurabo (Osaka, Japan) and were cultured in HuMedia-KB2 (Kurabo) with Human Keratinocyte Growth Supplement (Invitrogen). To determine the effect of S100A7 on cell growth, Saos-2 cells were inoculated at $1 \times 10^{5}$ cells/well and cultured in a serum-free medium containing indicated recombinant proteins $(1 \mu \mathrm{g} / \mathrm{ml})$. The $10 \%$ FBS-containing medium was used as a positive control.

Preparation of recombinant proteins. S100A7 (NM002963) cDNA was amplified by PCR and cloned into pGEX6P1 (GE Healthcare Bio-Sciences, Piscataway, NJ, USA). The nucleotide sequence of the cDNA was confirmed by DNA sequencing. Escherichia coli (BL21) cells were transformed by the vector and the recombinant GST-fusion protein was purified by glutathione-agarose affinity chromatography using a Sepharose 4B column (GE Healthcare Bio-Sciences) under conventional conditions. GST was released by cleaving with PreScission protease (GE Healthcare Bio-Sciences) and removed from the final preparations using the Sepharose 4B column. Recombinant GST protein was also prepared and used as a negative control.

Pull-down assay and western blot analysis. Biotinylated recombinant S100A7 or GST protein $(5 \mu \mathrm{g})$ was incubated with $5 \mu \mathrm{g}$ of human RAGE Fc (R\&D Systems, Minneapolis, MN, USA) and the complex of proteins was pulled down using streptavidine agarose (Invitrogen). After washing the agarose beads, the bound proteins were eluted and fractionated by SDS-PAGE. Streptavidine-HRP (R\&D Systems) was used to detect the applied biotinylated S100A7 and GST proteins. Bound RAGE protein was detected by western blotting. Western blot analysis was performed under conventional conditions using $10 \mu \mathrm{g}$ of protein extracts (15). The antibodies used were as follows: mouse anti-RAGE antibody (R\&D Systems), mouse anti-tubulin antibody (Sigma, St. Louis, MO, USA) and HRP-linked anti-mouse IgG (Cell Signaling Technologies, Beverly, MA, USA).

RNA interference. siRNA against human RAGE (siGENOME SMART pool siRNA targeting AGER, Thermo Scientific Dharmacon, Lafayette, CO, USA) was transfected into cells using RNAi MAX reagent (Invitrogen). A control siRNA with no known mammalian homology (siGENONE non-targeting siRNA 1, Thermo Scientific Darmacon) was used as a negative control. The cells were incubated for $72 \mathrm{~h}$ and used for various assays.

Transmigration assay and invasion assay. The transmigration and invasion potentials of the cells were assayed in vitro under conditions similar to those described previously (16). Briefly, 50,000 osteosarcoma cells were inoculated into top wells of pre-coated Boyden chambers (pore size, $8 \mu \mathrm{m}$; BD Biosciences, Bedford, MA, USA). Following incubation, the filters were

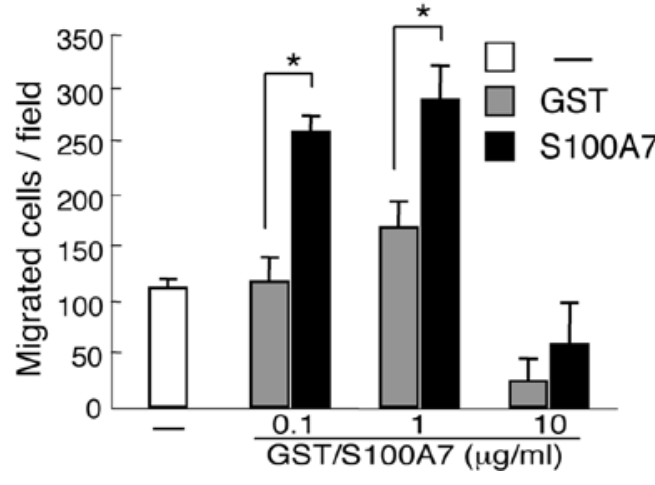

Figure 1. S100A7 promoted the migration of Saos-2 cells as assessed by a transmigration assay. Saos-2 cells $(50,000 /$ well $)$ were seeded into a Boyden chamber with purified recombinant proteins and cells that migrated were counted after 24 h. ${ }^{*} \mathrm{P}<0.05$.

stained and the cells on the bottom surface were counted. For the transmigration assay, the chambers were coated with $2 \mu \mathrm{g}$ of human fibronectin (Sigma) on the bottom and the incubation time was $8 \mathrm{~h}$. For the invasion assay, $2 \mu \mathrm{g}$ of human fibronectin and $10 \mu \mathrm{g}$ of Matrigel (BD Biosciences) were applied onto the upper and lower sides, respectively, and the cells were incubated for $24 \mathrm{~h}$. Mean values were obtained from 10 visual fields.

Gelatin zymography. Saos-2 cells were treated with siRNA for $24 \mathrm{~h}$, washed twice and incubated with $10 \mathrm{ml}$ of Opti-MEM (Invitrogen) supplemented with either S100A7 or GST. The conditioned medium was collected and concentrated into $100 \mu 1$ by centrifugation using a Centricon (Amicon Ultra-15 Ultracell-10k; Millipore, Billerica, MA, USA) and then mixed with $50 \mu \mathrm{l}$ of $3 \mathrm{X}$ SDS sample buffer. The samples $(10 \mu \mathrm{l})$ were applied onto an $8 \%$ SDS-polyacrylamide slab gel containing $0.5 \%$ gelatin (Sigma). Following electrophoresis, the gel was washed with water to remove the SDS, soaked in protein refolding buffer $(2.5 \%$ Triton X-100, $10 \%$ glycerol, $0.5 \mathrm{mM}$ $\mathrm{CaCl}_{2}, 100 \mathrm{mM} \mathrm{NaCl}, 50 \mathrm{mM}$ Tris-HCl$/ \mathrm{pH}$ 7.4) for $1 \mathrm{~h}$ and incubated in $50 \mathrm{mM}$ Tris- $\mathrm{HCl} / \mathrm{pH} 7.4$ containing $0.5 \mathrm{mM} \mathrm{CaCl}_{2}$ for $12 \mathrm{~h}$ at $37^{\circ} \mathrm{C}$. The gel was then stained with $1 \%$ Coomassie brilliant blue (CBB) and further treated with $10 \%$ methanol and $5 \%$ acetic acid to destain it. The gelatinolytic activity was detected as clear bands on a blue background of the CBB-stained gel. The recombinant matrix metalloproteinase (MMP)-2 and -9 proteins (R\&D Systems) were used as positive controls.

Statistical analysis. Each experiment was repeated a minimum of three times. The results are expressed as the mean $\pm \mathrm{SD}$. The Student's t-test was used to compare the two groups. $\mathrm{P}<0.05$ was considered to indicate a statistically significant result.

\section{Results and Discussion}

S100A7 promoted the migration of Saos-2 cells. We screened for S100 proteins that promote the migration of osteosarcoma cells. The cDNA of $20 \mathrm{~S} 100$ family members was cloned and GST-fused recombinant proteins were prepared. The proteins were applied to Saos-2 cells in the wound migration assay in vitro. Among the member proteins, S100A7 was identified as the most promising candidate (data not shown). 


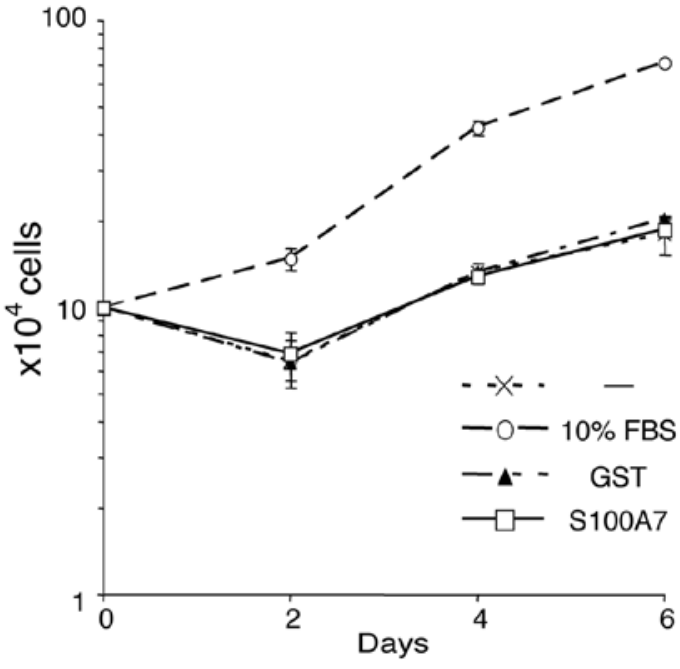

Figure 2. Effect of S100A7 on cell growth of Saos-2 osteosarcoma. Saos-2 cells were cultured in a serum-free medium containing $10 \%$ FBS or $1 \mu \mathrm{g} / \mathrm{ml}$ recombinant proteins (GST or S100A7).
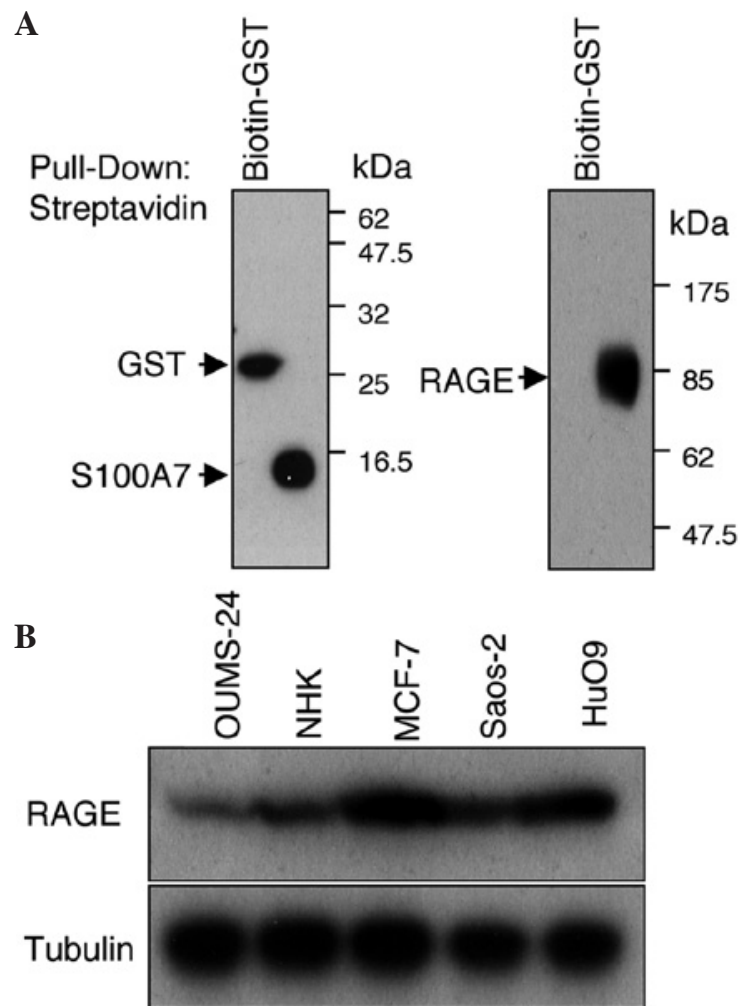

Figure 3. Direct binding of S100A7 with RAGE. (A) A pull-down assay using recombinant proteins. Biotinylated GST or GST-S100A7 $(5 \mu \mathrm{g})$ was incubated with human RAGE Fc, pulled down using streptavidin-agarose and eluted. Left panel: GST and GST-S100A7 were visualized using streptabidineHRP. Right panel: RAGE protein that co-precipitated with GST-S100A7 was detected by anti-RAGE antibody. (B) Expression of RAGE in normal fibroblasts (OUMS-24), normal human keratinocytes (NHK), breast cancer cells (MCF-7) and osteosarcoma cells (Saos-2 and HuO9) as demonstrated by western blot analysis.

We confirmed the function of S100A7 in Saos-2 cells by the transmigration assay (Fig. 1). The number of cells that migrated was significantly increased by the S100A7 protein and the extent of the increase was dose-dependent. However,
A

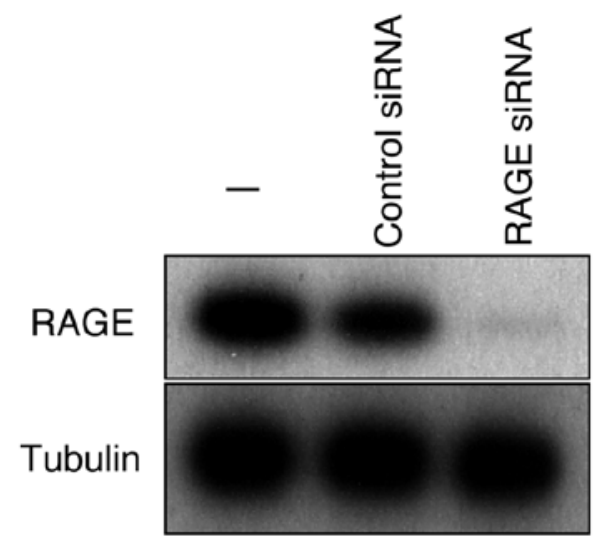

B

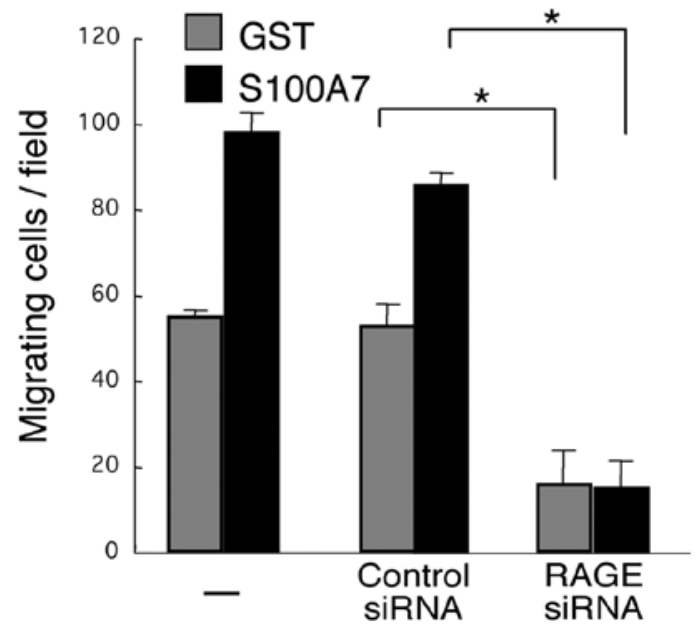

Figure 4. Inhibition of the migration of Saos-2 cells by the downregulation of RAGE. (A) Extracts were analyzed by western blotting $72 \mathrm{~h}$ after the application of 150 pmol RAGE siRNA to Saos-2 cells. (B) Cells in which RAGE was downregulated were used for a 24 -h transmigration assay. ${ }^{*} \mathrm{P}<0.05$.

the growth of the Saos- 2 cells was not affected by the addition of S100A7 protein (Fig. 2).

RAGE was necessary in S100A7-dependent promotion of Saos-2 cell migration. S100 proteins are recognized as damageassociated molecular pattern proteins (DAMPs) due to their release from damaged cells under conditions of cell stress (17). Certain DAMPs, including S100 proteins, are thought to be ligands for the multiligand receptor RAGE. RAGE is a type I transmembrane protein belonging to the immunoglobulin superfamily (18) and is involved in a broad range of inflammatory, degenerative and hyperproliferative diseases, including sepsis, rheumatoid arthritis, diabetic nephropathy/angiopathy, atherosclerosis, cancer and neurological disorders $(19,20)$. The receptor is composed of an extracellular region, a hydrophobic transmembrane-spanning domain and a short cytoplasmic tail. The extracellular domain binds a number of ligands, including advanced glycation end products, high-mobility group box 1 , S100 family proteins and amyloid fibrils (21). Wolf et al (9) revealed the interaction between S100A7 and RAGE by a competitive ligand binding assay. We performed a pull-down assay using recombinant RAGE and biotinylated S100A7 proteins (Fig. 3A). The RAGE protein was co-precipitated with biotinylated GST-S100A7 but not with biotinylated GST. The measurement of the surface plasmon resonance 
A

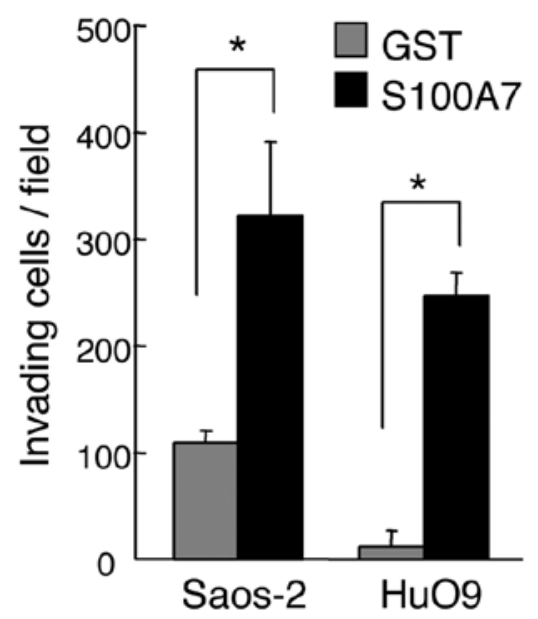

B

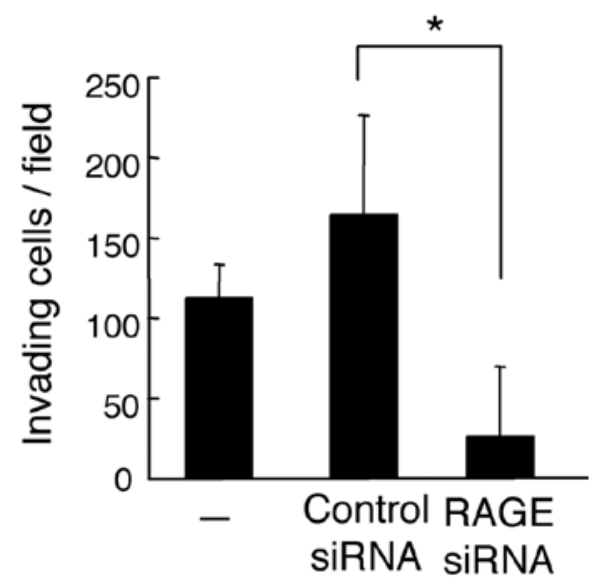

C

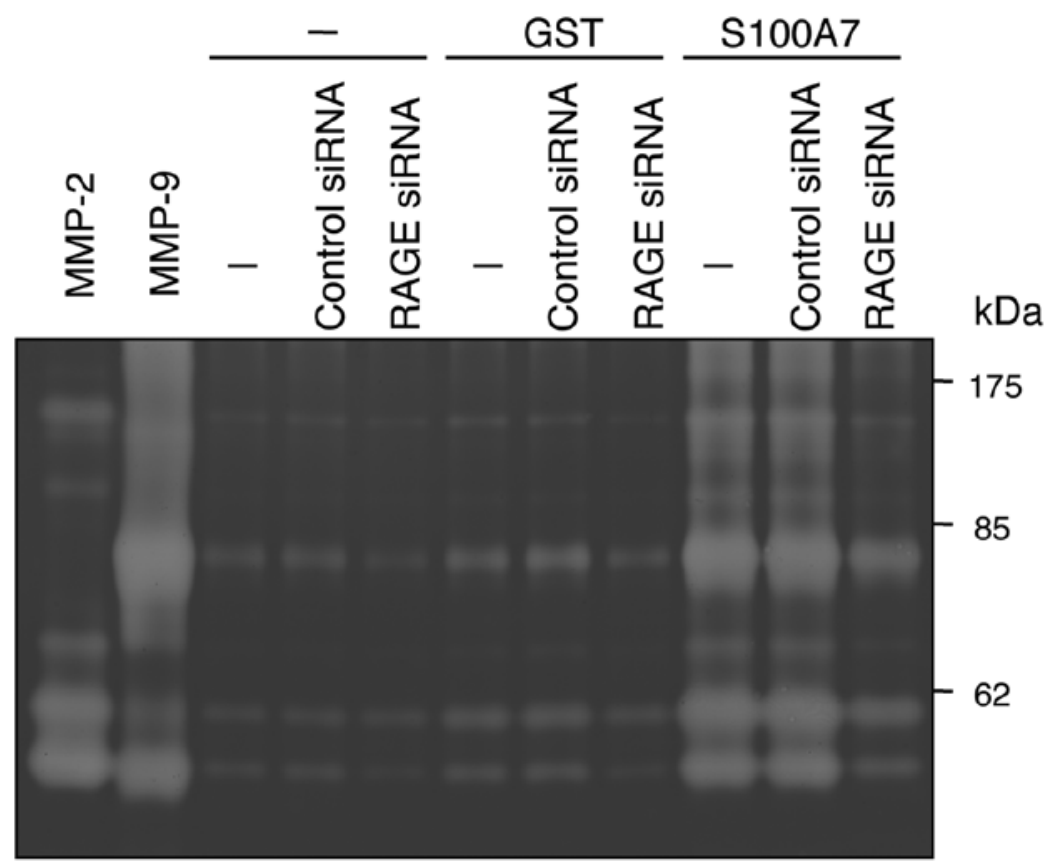

Figure 5. Effect of S100A7 protein on the invasion of osteosarcoma cells. (A) Enhanced invasion of Saos-2 and HuO9 cells by S100A7. The experiment was performed under conditions similar to those in Fig. 1, with the exception of coating the upper side of the membrane with Matrigel. The concentration of the proteins was $1 \mu \mathrm{g} / \mathrm{ml}$. " $\mathrm{P}<0.05$ (B) Downregulation of RAGE inhibited the invasion of Saos-2 cells as assayed in the presence of $1 \mu \mathrm{g} / \mathrm{ml} \mathrm{S} 100 \mathrm{~A} 7$. siRNA was applied to the cells under conditions similar to those in Fig. 3. "P $<0.05$ (C) Gelatin zymography of Saos- 2 cells, which were processed under conditions identical to those in (B). The cells were subjected to gelatin zymography $24 \mathrm{~h}$ after incubation with purified recombinant proteins in the serum-free medium.

of these proteins also supported their interaction (data not shown). RAGE is expressed in various types of human cancer, including brain, breast, colon, lung, oral squamous cell and ovarian cancer (22). The expression of RAGE was examined in osteosarcoma cell lines, and the results showed that compared with normal fibroblasts and keratinocytes, which are known to express RAGE protein, osteosarcoma cells (Saos-2 and HuO9) expressed RAGE protein at high levels (Fig. 3B).

The functional involvement of RAGE in the S100A7induced promotion of migration of osteosarcoma cells was also assessed. The expression of RAGE was successfully downregulated by the specific siRNA in Saos-2 cells (Fig. 4A). The downregulation of RAGE resulted in marked eradication of the S100A7-promoted transmigration of Saos-2 cells (Fig. 4B). Notably, RAGE downregulation suppressed not only the migration of S100A7-stimulated Saos-2 cells but also that of unstimulated cells, suggesting a potential role of endogenous S100A7 and/or other RAGE ligands in transmigration capacity.

S100A7 promoted the invasion and MMP activity of osteosarcoma cells. The coordination of cell migration and the degradation of matrix proteins is crucial for the invasion of malignant cells. We therefore performed an invasion assay that mimicks the invasion of cancer cells passing through the basement membrane (23). The addition of S100A7 significantly increased the invasion capacity of Saos-2 and HuO9 osteosarcoma cells (Fig. 5A). The downregulation of RAGE by siRNA demonstrates that the invasion of Saos-2 cells also depends on the expression of RAGE (Fig. 5B).

MMPs are known to be involved in the invasion of various types of cancer cells, including osteosarcoma cells $(24,25)$. We analyzed the activity of MMPs by gelatin zymography 
(Fig. 5C). The application of S100A7 to Saos-2 cells resulted in a marked enhancement of the activity of MMP-2 and MMP-9 (Fig. 5C). Collectively, our results indicate that the S100A7-RAGE signal transdution pathway is involved in the invasion of osteosarcoma cells.

$\mathrm{S} 100 \mathrm{~A} 7$ is known to promote breast cancer progression (26). However, S100A7 has been reported to act as a negative regulator of breast cancer invasion (27). Our data have demonstrated that S100A7 promoted the migration and invasion of osteosarcoma cells in vitro. A crucial question is whether S100A7 functions to promote the invasion and/or metastasis of osteosarcoma in vivo. Hiratsuka et al (28) revealed that S100A8 and S100A9 indirectly act on the lung to accelerate the migration of primary tumor cells to lung tissues. Andresen et al (29) reported that the expression of S100A7 was detected in the normal lung and that it was increased under pathological conditions. An increased production of S100A7 in the lung may enable circulating cancer cells to settle and grow invasively in the lung. Although further studies are needed, the S100A7-RAGE axis may be a new target for preventing the invasion and/or metastasis of osteosarcoma.

\section{Acknowledgements}

This study was supported in part by grants from the Ministry of Education, Culture, Sports, Science, and Technology of Japan (70379840 to M. Sakaguchi and 21591699 to K. Kataoka).

\section{References}

1. Longhi A, Errani C, De Paolis M, Mercuri M and Bacci G Primary bone osteosarcoma in the pediatric age: state of the art Cancer Treat Rev 32: 423-436, 2006.

2. Provisor AJ, Ettinger LJ, Nachman JB, Krailo MD, Makley JT, Yunis EJ, Huvos AG, Betcher DL, Baum ES, Kisker CT and Miser JS: Treatment of nonmetastatic osteosarcoma of the extremity with preoperative and postoperative chemotherapy: a report from the Children's Cancer Group. J Clin Oncol 15: 76-84, 1997.

3. Ferguson WS and Goorin AM: Current treatment of osteosarcoma. Cancer Invest 19: 292-315, 2001.

4. Eckert RL, Broome AM, Ruse M, Robinson N, Ryan D and Lee K: S100 proteins in the epidermis. J Invest Dermatol 123: 23-33, 2004.

5. Donato R: Intracellular and extracellular roles of S100 proteins. Microsc Res Tech 60: 540-551, 2003.

6. Salama I, Malone PS, Mihaimeed F and Jones JL: A review of the S100 proteins in cancer. Eur J Surg Oncol 34: 357-364, 2008.

7. Boye K and Maelandsmo GM: S100A4 and metastasis: a small actor playing many roles. Am J Pathol 176: 528-535, 2010.

8. Madsen P, Rasmussen HH, Leffers H, Honoré B, Dejgaard K, Olsen E, Kiil J, Walbum E, Andersen AH, Basse B, et al: Molecular cloning, occurrence, and expression of a novel partially secreted protein 'psoriasin' that is highly up-regulated in psoriatic skin. J Invest Dermatol 97: 701-712, 1991.

9. Wolf R, Howard OM, Dong HF, Voscopoulos C, Boeshans K, Winston J, Divi R, Gunsior M, Goldsmith P, Ahvazi B, et al: Chemotactic activity of S100A7 (Psoriasin) is mediated by the receptor for advanced glycation end products and potentiates inflammation with highly homologous but functionally distinct S100A15. J Immunol 181: 1499-1506, 2008.
10. EngelkampD, Schäfer BW, Mattei MG,Erne P and Heizmann CW: Six S100 genes are clustered on human chromosome 1q21: identification of two genes coding for the two previously unreported calcium-binding proteins S100D and S100E. Proc Natl Acad Sci USA 90: 6547-6551, 1993.

11. Rodan SB, Imai Y, Thiede MA, Wesolowski G, Thompson D, Bar-Shavit Z, Shull S, Mann K and Rodan GA: Characterization of a human osteosarcoma cell line (Saos-2) with osteoblastic properties. Cancer Res 47: 4961-4966, 1987.

12. Soule HD, Vazguez J, Long A, Albert S and Brennan M: A human cell line from a pleural effusion derived from a breast carcinoma. J Natl Cancer Inst 51: 1409-1416, 1973.

13. Bai L, Mihara K, Kondo Y, Honma M and Namba M: Immortalization of normal human fibroblasts by treatment with 4-nitroquinoline 1-oxide. Int J Cancer 53: 451-456, 1993.

14. Kawai A, Ozaki T, Ikeda S, Oda T, Miyazaki M, Sato J, Taketa K and Inoue H: Two distinct cell lines derived from a human osteosarcoma. J Cancer Res Clin Oncol 115: 531-536, 1989.

15. Yamamoto K, Sakaguchi M, Medina RJ, Niida A, Sakaguchi Y, Miyazaki M, Kataoka K and Huh NH: Transcriptional regulation of a brown adipocyte-specific gene, UCP1, by KLF11 and KLF15. Biochem Biophys Res Commun 400: 175-180, 2010.

16. Kataoka K and Huh NH: A novel beta1,3-N-acetylglucosaminyltransferase involved in invasion of cancer cells as assayed in vitro. Biochem Biophys Res Commun 294: 843-848, 2002.

17. Foell D, Wittkowski H, Vogl T and Roth J: S100 proteins expressed in phagocytes: a novel group of damage-associated molecular pattern molecules. J Leukoc Biol 81: 28-37, 2007.

18. Neeper M, Schmidt AM, Brett J, Yan SD, Wang F, Pan YC, Elliston K, Stern D and Shaw A: Cloning and expression of a cell surface receptor for advanced glycosylation end products of proteins. J Biol Chem 267: 14998-15004, 1992.

19. Yan SF, Ramasamy R and Schmidt AM: Mechanisms of disease: advanced glycation end-products and their receptor in inflammation and diabetes complications. Nat Clin Pract Endocrinol Metab 4: 285-293, 2008.

20. Rojas A, Figueroa $\mathrm{H}$ and Morales E: Fueling inflammation at tumor microenvironment: the role of multiligand/RAGE axis. Carcinogenesis 31: 334-341, 2010.

21. Sparvero LJ, Asafu-Adjei D, Kang R, Tang D, Amin N, Im J, Rutledge R, Lin B, Amoscato AA, Zeh HJ and Lotze MT: RAGE (Receptor for Advanced Glycation Endproducts), RAGE ligands, and their role in cancer and inflammation. J Transl Med 7: 17, 2009.

22. Riehl A, Németh J, Angel P and Hess J: The receptor RAGE: Bridging inflammation and cancer. Cell Commun Signal 7: 12, 2009.

23. Albini A and Benelli R: The chemoinvasion assay: a method to assess tumor and endothelial cell invasion and its modulation. Nat Protoc 2: 504-511, 2007.

24. Gialeli C, Theocharis AD and Karamanos NK: Roles of matrix metalloproteinases in cancer progression and their pharmacological targeting. FEBS J 278: 16-27, 2011.

25. Xin ZF, Kim YK and Jung ST: Risedronate inhibits human osteosarcoma cell invasion. J Exp Clin Cancer Res 28: 105, 2009.

26. Emberley ED, Murphy LC and Watson PH: S100A7 and the progression of breast cancer. Breast Cancer Res 6: 153-159, 2004

27. Krop I, März A, Carlsson H, Li X, Bloushtain-Qimron N, Hu M, Gelman R, Sabel MS, Schnitt S, Ramaswamy S, et al: A putative role for psoriasin in breast tumor progression. Cancer Res 65 : 11326-11334, 2005.

28. Hiratsuka S, Watanabe A, Sakurai Y, Akashi-Takamura S, Ishibashi S, Miyake K, Shibuya M, Akira S, Aburatani $\mathrm{H}$ and Maru Y: The S100A8-serum amyloid A3-TLR4 paracrine cascade establishes a pre-metastatic phase. Nat Cell Biol 10: 1349-1355, 2008.

29. Andresen E, Lange C, Strodthoff D, Goldmann T, Fischer N, Sahly H, Branscheid D and Heine H: S100A7/psoriasin expression in the human lung: unchanged in patients with COPD, but upregulated upon positive $S$. aureus detection. BMC Pulm Med 11: 10, 2011. 